\title{
Pseudogap and Vortices in High-Temperature Superconductors
}

\author{
M. MAŚKA AND M. MiERZEJEWSKI \\ Department of Theoretical Physics, Institute of Physics, Silesian University \\ Uniwersytecka 4, 40-007 Katowice, Poland
}

\begin{abstract}
The origin of the pseudogap is one of the most puzzling features of the high-temperature superconductors. There are two main scenarios: the first one assumes the presence of a hidden order competing or coexisting with superconductivity; within the framework of the second one the pseudogap is a precursor of the superconducting gap. In this paper we present some aspects of the hidden order pseudogap scenario. In particular, we discuss how the competing order modifies the structure of vortices in high-temperature superconductors. We demonstrate that the presence of the hidden order can explain some features of vortices observed in scanning tunneling microscopy experiments.
\end{abstract}

PACS numbers: 74.25.Ha, 74.25.Op, 74.20.--z

\section{Introduction}

The pseudogap that opens in the normal state of cuprates has recently been the central problem in the physics of high- $T_{\mathrm{c}}$ superconductivity. The presence of the pseudogap has been confirmed with the help of various experimental techniques like: angle-resolved photoemission, intrinsic tunneling spectroscopy, NMR, infrared and transport measurements. Although there is no generally accepted theoretical approach to the pseudogap, one usually considers this phase as a precursor of the superconductivity. Within this scenario formation of the Cooper pairs starts at temperature $T^{*}$ that is higher than the superconducting transition temperature $T_{\mathrm{c}}$. Then, at $T_{\mathrm{c}}$, these preformed pairs undergo the Bose-Einstein condensation. This hypothesis seems to be supported by recent observations of the vortex-like Nernst signal above $T_{\mathrm{c}}[1]$ that evolves smoothly into the analogous signal below the superconducting phase transition [2]. 
On the other hand, the pseudogap may represent other phase unrelated to superconductivity. Competition between these phases allows one to explain qualitatively the nonmonotonic doping dependence of $T_{\mathrm{c}}[3]$. Within this approach it is obvious that increase in $T^{*}$ corresponds to decrease in $T_{\mathrm{c}}$, as it is observed in underdoped high-temperature superconductors (HTSC). Aside from other approaches, a $d$-density-wave (DDW) state has recently been proposed as an order that competes with superconductivity [3-5]. According to this hypothesis the pseudogap opens due to a condensation of electron-hole pairs with angular momentum $l=2$. As a result, there occur orbital currents, which alter from one lattice bond to a neighboring one. The DDW state breaks parity as well as translational, rotational, and time reversion symmetries. As the DDW and superconducting orders compete [3], one may expect that reduction of one of them may lead to a strong enhancement of the other. The DDW order is almost insensitive to the magnetic field [6], and therefore application of external magnetic field seems to be a natural tool to carry out this procedure. In particular, one may expect an enhancement of the DDW gap in the vortex core, where the superconductivity is suppressed. In the present paper we investigate the vortex structure assuming the DDW scenario of the pseudogap. Recent developments of the scanning tunneling microscopy (STM) give a new insight into the electronic structure of the vortex cores and allow one to directly compare the experimental data with theoretical predictions presented here. In particular, we show that the hidden order scenario explains the tunneling spectra obtained in the vicinity of the vortex core in BSCCO [7].

\section{Model}

We start with a mean-field Hamiltonian that describes a system with coexisting/competing DDW and $d$-wave superconductivity (DSC)

$$
H=-t \sum_{\langle i j\rangle \sigma} \mathrm{e}^{\mathrm{i} \theta_{i j}} a_{i \sigma}^{\dagger} a_{j \sigma}-\mu \sum_{i \sigma} a_{i \sigma}^{\dagger} a_{i \sigma}+H^{\prime},
$$

where

$$
H^{\prime}=\sum_{\langle i j\rangle}\left(a_{i \uparrow}^{\dagger} a_{j \downarrow}^{\dagger} \Delta_{i j}+a_{i \downarrow} a_{j \uparrow} \Delta_{i j}^{*}\right)+\sum_{\langle i j\rangle \sigma}(-1)^{i} D_{i j} \mathrm{e}^{\mathrm{i} \theta_{i j}} a_{i \sigma}^{\dagger} a_{j \sigma},
$$

and $t$ is the nearest-neighbor hopping integral in the absence of the external magnetic field. The magnetic field is introduced through the Peierls phase factor $\mathrm{e}^{\mathrm{i} \theta_{i j}}$, that is responsible for the diamagnetic response of the system: $\theta_{i j}=\frac{e}{\hbar c} \int_{\boldsymbol{R}_{j}}^{\boldsymbol{R}_{i}} \boldsymbol{A} \cdot \mathrm{d} \boldsymbol{l}$. We assume that the magnetic field is uniform, which is justified for extremely type II superconductors. The first term in Eq. (2) is the nearest-neighbor pairing that leads to anisotropic superconductivity of $d$-wave symmetry

$$
\Delta_{i j}=-\frac{V_{\mathrm{DSC}}}{2}\left\langle a_{i \downarrow} a_{j \uparrow}-a_{i \uparrow} a_{j \downarrow}\right\rangle
$$

whereas the second one is responsible for DDW state with the order parameter 
given by

$$
D_{i j}=(-1)^{i} \frac{V_{\mathrm{DDW}}}{2}\left\langle\mathrm{e}^{\mathrm{i} \theta_{i j}} a_{i \sigma}^{\dagger} a_{j \sigma}-\mathrm{e}^{\mathrm{i} \theta_{j i}} a_{j \sigma}^{\dagger} a_{i \sigma}\right\rangle .
$$

In order to diagonalize the Hamiltonian (1), we introduce new fermionic operators $\gamma_{n \sigma}$,

$$
a_{i \uparrow}=\sum_{l} u_{i l} \gamma_{l \uparrow}-v_{i l}^{*} \gamma_{l \downarrow}^{\dagger}, \quad a_{i \downarrow}=\sum_{l} u_{i l} \gamma_{l \downarrow}+v_{i l}^{*} \gamma_{l \uparrow}^{\dagger},
$$

where $u_{i l}$ and $v_{i l}$ are determined by the Bogoliubov-de Gennes (BdG) equations

$$
\sum_{j}\left(\begin{array}{cc}
\mathcal{H}_{i j} & \Delta_{i j} \\
\Delta_{i j}^{*} & -\mathcal{H}_{i j}^{*}
\end{array}\right)\left(\begin{array}{c}
u_{j l} \\
v_{j l}
\end{array}\right)=E_{l}\left(\begin{array}{c}
u_{i l} \\
v_{i l}
\end{array}\right)
$$

with the single particle Hamiltonian given by

$$
\mathcal{H}_{i j}=\left[-t \delta_{i+\delta, j}+(-1)^{i} D_{i j}\right] \mathrm{e}^{\mathrm{i} \theta_{i j}}-\mu \delta_{i j} .
$$

Then, the Hamiltonian expressed in terms of the new operators takes on the following form:

$$
H=\sum_{l \sigma} E_{l} \gamma_{l \sigma}^{\dagger} \gamma_{l \sigma}+\text { const. }
$$

In the next step, we calculate the DDW and DSC order parameters

$$
\begin{aligned}
D_{i j} & =(-1)^{i} \frac{\mathrm{i} V_{\mathrm{DDW}}}{2} \sum_{l} \operatorname{Im}\left(u_{i l} u_{j l}^{*} \mathrm{e}^{\mathrm{i} \theta_{i j}}+v_{i l} v_{j l}^{*} \mathrm{e}^{-\mathrm{i} \theta_{i j}}\right) \tanh \left(\frac{E_{l}}{2 k T}\right), \\
\Delta_{i j} & =\frac{V_{\mathrm{DSC}}}{2} \sum_{l}\left(u_{i l} v_{j l}^{*}+v_{i l}^{*} u_{j l}\right) \tanh \left(\frac{E_{l}}{2 k T}\right),
\end{aligned}
$$

as well as the local concentration of carriers

$$
n_{i}=2 \sum_{l}\left|u_{i l}\right|^{2} f\left(E_{l}\right)+\left|v_{i l}\right|^{2} f\left(-E_{l}\right) .
$$

Equation (6) represents a standard eigenvalue problem. However, the elements of the diagonalized matrix explicitly depend on eigenvalues and eigenvectors and, therefore, the diagonalization should be carried out self-consistently with Eqs. (9), (10) and (11). In order to compare the numerical results with STM data we also calculate the local density of states (LDOS),

$$
\rho_{i}(\epsilon)=-\sum_{l}\left[\left|u_{i l}\right|^{2} f^{\prime}\left(E_{l}-\epsilon\right)+\left|v_{i l}\right|^{2} f^{\prime}\left(E_{l}+\epsilon\right)\right] .
$$

This quantity is proportional to the local differential tunneling conductance that could be measured in STM experiments. 


\section{Results and discussion}

The BdG equations were solved numerically for $35 \times 35$ lattice. We took the nearest-neighbor hopping integral as the energy unit and assumed $V_{\mathrm{DDW}}=1.6, V_{\mathrm{DSC}}=1.4$. In the absence of magnetic field the phase diagram of HTSC can qualitatively be reproduced for such values of the interaction strengths [8] (see the lower part in Fig. 1).

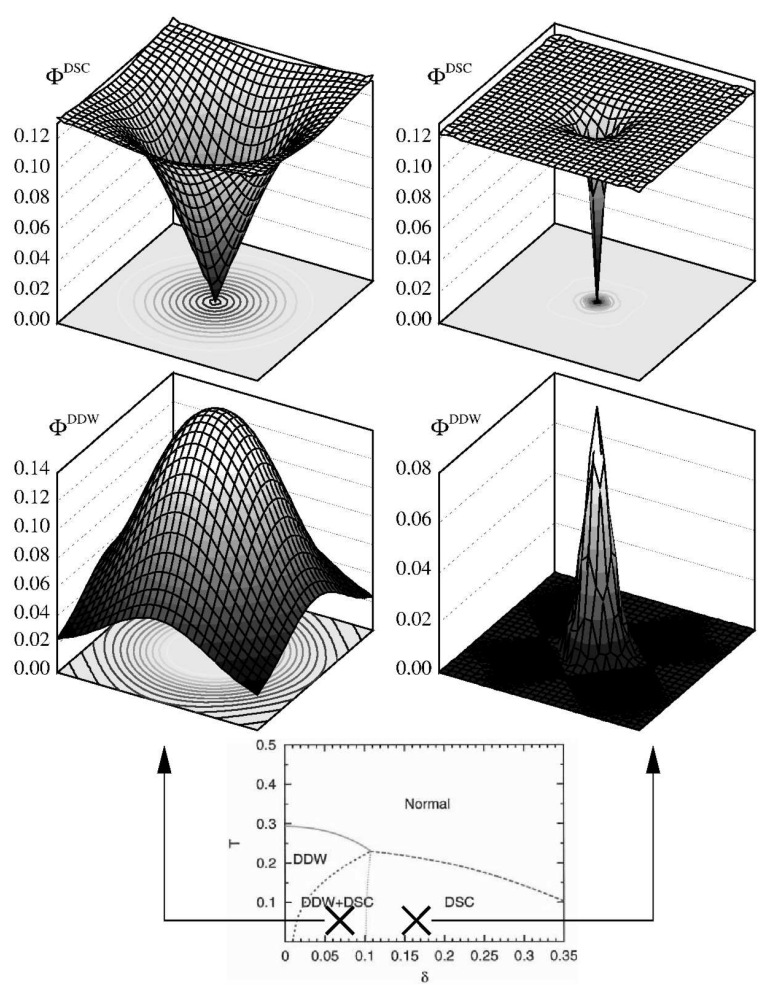

Fig. 1. The DDW and DSC order parameters in the vicinity of the vortex core. Left and right parts correspond to different concentration of holes $(\delta)$, indicated by crosses in the lower part. This part shows a phase diagram in the absence of magnetic field, taken from Ref. [8]. Reprinted figure with permission from J.X. Zhu, W. Kim, C.S. Ting, J.P. Carbotte, Phys. Rev. Lett. 87, 197001 (2001). Copyright (2001) by the American Physical Society.

Figure 1 shows typical solutions of underdoped and overdoped systems. In the first case both the orders coexist in the homogeneous regions. However, in the vicinity of the vortex core DSC order vanishes. This, in turn, is responsible for a strong enhancement of the competing order (see the left part in Fig. 1). In the overdoped regime DDW does not occur in the absence of magnetic field. However, 
in the vortex core this phase may set on, provided doping is not too large (the right part in Fig. 1). We have found that $\left|\Delta_{i j}\right|^{2}+\left|D_{i j}\right|^{2}$ is almost spatially uniform [9], which clearly demonstrates the competition between both the orders.

This mechanism is of crucial importance for LDOS in the vortex core. In the absence of DDW one expects that the zero bias conductance peak (ZBCP) should appear in the vortex core. However, development of the DDW gap in the core strongly reduces ZBCP. The pure DDW gap evolves smoothly into DSC or DSC+DDW gaps when the STM tip moves away from the vortex center (see Fig. 2). However, DSC and DDW gaps differ in the sense that the first one opens at the Fermi level, whereas the second one occurs in the middle of the band. This feature is responsible for the asymmetry of the LDOS inside the vortex. In particular, the peak at positive bias shifts outwards when approaching the center of vortex, whereas the peak at negative bias does not move. Such an asymmetry has recently been observed in BSCCO [7]. For larger doping this feature is even more pronounced, since there is no DDW gap away from the vortex and the Fermi level is much below the center of the band.
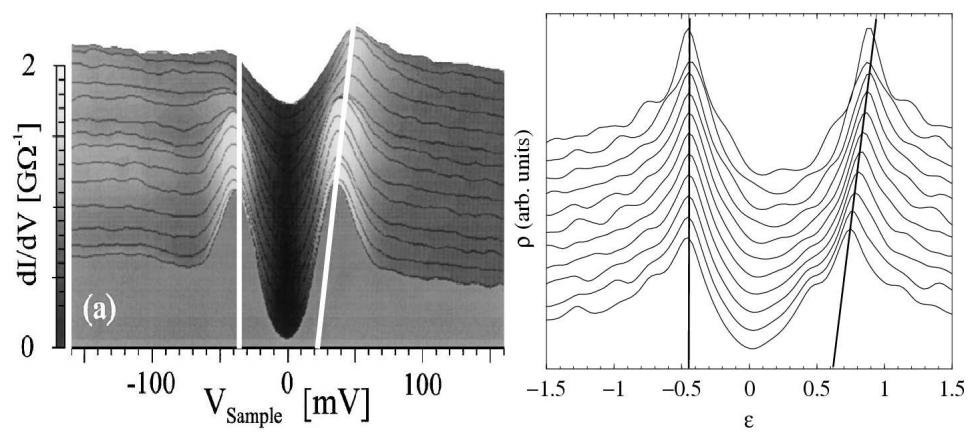

Fig. 2. Asymmetry of the LDOS in the vortex core. The left part shows experimental results taken from Ref. [7], whereas the right one shows our numerical results. Reprinted figure with permission from Ch. Renner, B. Revaz, K. Kadowaki, I. Maggio-Aprile, Ø. Fischer, Phys. Rev. Lett. 80, 3606 (1998). Copyright (1998) by the American Physical Society.

It was shown in 1964 by Caroli et al. [10] that there should be bound states around the vortex core in an isotropic $s$-wave superconductor. These states manifest themselves in LDOS as two symmetric peaks near the Fermi surface. It was later explained by Franz and Tesanovič that there are no truly bound states in $d$-wave superconductors and all the states are extended with continuous energy spectrum [11]. On the other hand, Maggio-Aprile and others have recently found a splitting of the central peak in YBCO [12] and BSCCO [13]. As DDW and DSC gaps have nodes in the same directions, the core bound states do not occur in our approach. However, an additional component of the order parameter, for which the 

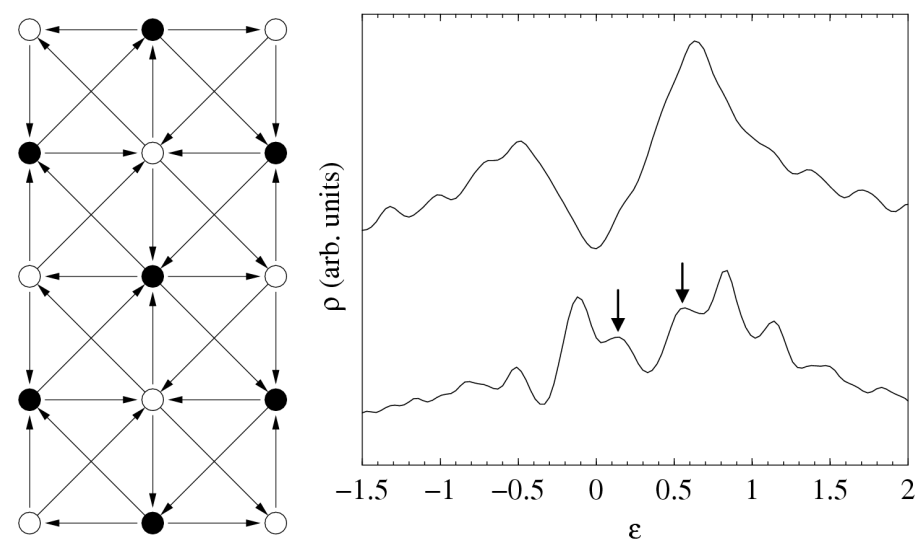

Fig. 3. The left part shows currents circulating in $d_{x^{2}-y^{2}}+\mathrm{i} d_{x y}$ density-wave state. In the right one we compare LDOS in the vortex center calculated with (lower curve) and without (upper curve) $d_{x y}$ component. Here, the arrows indicate the double-peak structure suggesting the presence of the bound states in the vortex core.

total gap is nodeless, leads to the splitting of the zero-bias peak in LDOS. Results presented in Fig. 3 demonstrate this case. Namely, we have considered $d_{x^{2}-y^{2}}+\mathrm{i} d_{x y}$ density wave. Such a state consists of staggered currents that flow between the nearest and next nearest neighboring sites. The resulting gap is nodeless and the double peak structure sets on, in accordance with the experimental data.

\section{References}

[1] Z.A. Xu, N.P. Ong, Y. Wang, T. Kakeshita, S. Uchida, Nature 406, 486 (2000).

[2] Yayu Wang, Z.A. Xu, T. Kakeshita, S. Uchida, S. Ono, Yoichi Ando, N.P. Ong, Phys. Rev. B 64, 224519 (2001).

[3] S. Chakravarty, R.B. Laughlin, D.K. Morr, C. Nayak, Phys. Rev. B 63, 094503 (2001).

[4] C. Nayak, Phys. Rev. B 62, 4880 (2000).

[5] S. Tewari, H.Y. Kee, C. Nayak, S. Chakravarty, Phys. Rev. B 64, 224516 (2001).

[6] H.K. Nguyen, S. Chakravarty, Phys. Rev. B 65, 180519 (2002).

[7] Ch. Renner, B. Revaz, K. Kadowaki, I. Maggio-Aprile, Ø. Fischer, Phys. Rev. Lett. 80, 3606 (1998).

[8] J.X. Zhu, W. Kim, C.S. Ting, J.P. Carbotte, Phys. Rev. Lett. 87, 197001 (2001).

[9] M. Maśka, M. Mierzejewski, Phys. Rev. B 68, 024513 (2003).

[10] C. Caroli, P.G. de Gennes, J. Matricon, Phys. Rev. Lett. 9, 307 (1964).

[11] M. Franz, Z. Tesanovic, Phys. Rev. Lett. 80, 4763 (1998).

[12] I. Maggio-Aprile, Ch. Renner, A. Erb, E. Walker, Ø. Fischer, Phys. Rev. Lett. 75, 2754 (1995).

[13] S.H. Pan, E.W. Hudson, A.K. Gupta, K.-W. Ng, H. Eisaki, S. Uchida, J.C. Davis, Phys. Rev. Lett. 85, 1536 (2000). 